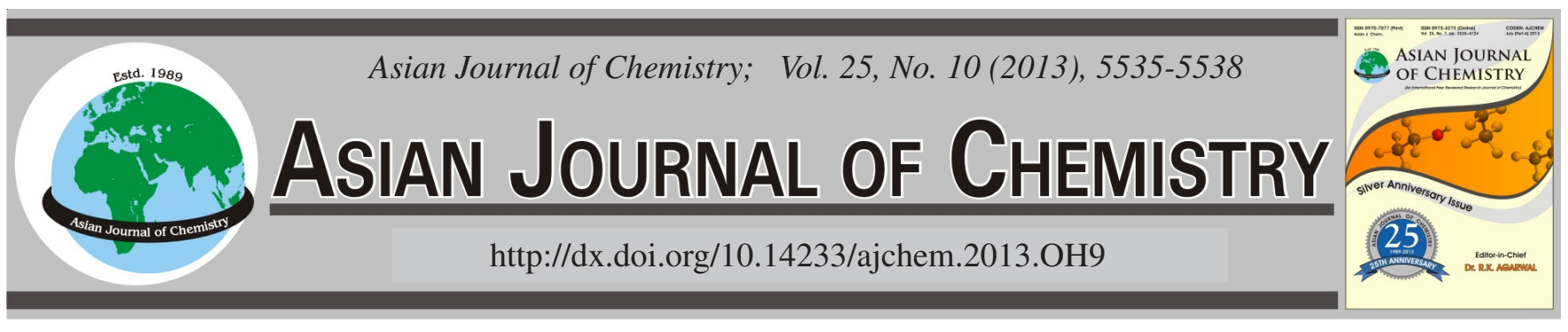

\title{
Microwave-assisted Synthesis of 2-Methyl-4-Quinolinones via Combes Synthesis Catalyzed by Acidic Resin under Solvent-Free Condition $\dagger$
}

\author{
SHIZHEN YuAN ${ }^{*}$, KeHUA ZHANG and JingJING XIA
}

Department of Chemistry, Anhui University of Architecture, Hefei 230022, Anhui Province, P.R. China

*Corresponding author: Fax: +86 551 3828106; Tel: +86 551 3828151; E-mail: yuanshzh3@ @otmail.com

A simple and efficient method for the synthesis of 2-methylquinolin-4(1H)-one derivatives using NKC-9-resin as reusable eco-friendly catalyst via Combes cyclization under solvent-free and microwave irradiation conditions is described. The quinolines were obtained in high yields and in short reaction times.

Key Words: Combes, Quinoline, Microwave irradiation, NKC-9, Solvent-free.

\section{INTRODUCTION}

It is known that quinoline derivatives are important compounds in the bioorganic and medicinal chemistry, constituting the structure of interesting pharmacological properties ${ }^{1}$. A large number of quinolines have been widely used as antimalarial ${ }^{2}$, antiasthmatic ${ }^{3}$ and antiviral agent ${ }^{4}$. In addition, quinolines are valuable synthons used for the preparation of nano- and mesostructures with enhanced electronic and photonic properties ${ }^{5}$.

There are several methods for the formation of the quinoline ring system such as Skraup ${ }^{6}, \mathrm{Knorr}^{7}$, Conrad-Limpach ${ }^{8}$, Doebner-von Miller ${ }^{9}$, Combes $^{10}$, Friedländer reactions ${ }^{11}$, etc. Among them, although the Friedländer reaction has been studied more, the Combes reaction is still one of the most simple and straightforward approaches for the synthesis of quinolines. Traditionally, the Combes reaction is carried out in organic solvents (e.g., ethanol, xylene and diphenyl ether) and heating by using $\mathrm{H}_{2} \mathrm{SO}_{4}$ as catalysts. Because of economical and environmental concerns, recently, the solid acids, such as In $\mathrm{Cl}_{3},{ }^{12} \mathrm{NaHSO}_{4},{ }^{13}$ used as catalysts for the Combes reaction has attracted much attention in synthesis of quinolines under solvent-free condition. However, most of the synthetic protocols reported so far suffer from high temperatures, prolonged reaction times, low yields, difficulty of separation and purification and unrenewable catalysts. Therefore, it is necessary to develop a simple, clean and efficient method for Combes reaction.

\section{EXPERIMENTAL}

All material from Aldrich and used directly as received and Flash chromatographic sheet employed was purchased from Anhui Liangchen Silicon Material Co. Ltd., The reactions were carried out in a multimode microwave oven (frequency: $2450 \mathrm{~Hz}$; maximal power: $1600 \mathrm{~W}$ ).

To a mixture of aromatic amines $(2 \mathrm{mmol})$, ethyl acetoacetate $(2.4 \mathrm{mmol})$ and resin (NKC-9, $100 \mathrm{mg}$ ) was added. The mixture was homogenized with the help of glass rod at room temperature and irradiated in microwave oven $(400 \mathrm{~W})$ for 1.5 minutes by the interval of $10 \mathrm{sec}$. The reaction was monitored by TLC. After completion of the reaction, the mixture was extracted with $\mathrm{CH}_{2} \mathrm{Cl}_{2}$ and filtered, the organic phase was washed with saturated brine, dried over anhydrous magnesium sulfate and concentrated in vacuo. The pure products were obtained by flash chromatography on silica gel eluting with petroleum ether/EtOAc $(6: 1, \mathrm{v}: \mathrm{v})$ and identified by IR, ${ }^{1} \mathrm{H},{ }^{13} \mathrm{C}$ NMR and HRMS.

All melting points were determined on a WT-melting point apparatus and are uncorrected. IR (Perkin-Elmer, 2000 FTIR), ${ }^{1} \mathrm{H} \mathrm{NMR}\left(\mathrm{CDCl}_{3}, 300 \mathrm{MHz}\right),{ }^{13} \mathrm{C} \mathrm{NMR}\left(\mathrm{CDCl}_{3}, 75 \mathrm{MHz}\right)$ and MS-GC (HP5890 (II)/HP 5972, EI) spectra were obtained at the Center of Analytical Configuration of University of Science and Technology of China.

2-Methyl-1H-quinolin-4-one (3a): IR: 3439, 3035, 1648, 1602, 1572, 1476, 1340, $701 \mathrm{~cm}^{-1} ;{ }^{1} \mathrm{H}$ NMR (DMSO- $d_{6}, 300$ $\mathrm{MHz}) \delta: 2.14$ (s, 3H, $\left.\mathrm{CH}_{3}\right), 6.13$ (s, 1H, CH=), 7.21-7.48 (m, 4H, $\mathrm{Ph}), 8.36$ (s, $1 \mathrm{H}, \mathrm{NH}) ;{ }^{13} \mathrm{C}$ NMR (DMSO- $\left.d_{6}, 75 \mathrm{MHz}\right) \delta: 24.17$, 113.62, 120.26, 122.31, 124.33, 127.45, 134.20, 137.21, 152.40, 183.72; HRMS: calcd. for $\mathrm{C}_{10} \mathrm{H}_{9} \mathrm{NO}: 159.1845$, found: 159.3195 .

2,6-Dimethyl-1H-quinolin-4-one (3b): IR: 3342, 3032, $1647,1621,1574,1472,1342,731 \mathrm{~cm}^{-1} ;{ }^{1} \mathrm{H}$ NMR (DMSO- $d_{6}$, 
$300 \mathrm{MHz}) \delta: 2.23\left(\mathrm{~s}, 3 \mathrm{H}, \mathrm{CH}_{3}\right), 2.52\left(\mathrm{~s}, 3 \mathrm{H}, \mathrm{CH}_{3}\right), 6.09$ (s, $1 \mathrm{H}$, $\mathrm{CH}=), 7.24(\mathrm{~d}, J=7.6 \mathrm{~Hz}, 1 \mathrm{H}, \mathrm{Ph}), 7.53(\mathrm{~d}, J=7.5 \mathrm{~Hz}, 1 \mathrm{H}$, $\mathrm{Ph}), 7.78(\mathrm{~s}, 1 \mathrm{H}, \mathrm{Ph}), 9.10(\mathrm{~s}, 1 \mathrm{H}, \mathrm{NH}) ;{ }^{13} \mathrm{C}$ NMR (DMSO- $d_{6}$, $75 \mathrm{MHz}) \delta$ : 14.32, 24.28, 102.16, 116.53, 124.61, 127.11, 129.83, 133.74, 143.12, 153.62, 181.84.; HRMS: calcd. for $\mathrm{C}_{11} \mathrm{H}_{11} \mathrm{NO}: 173.2115$, found: 173.1892 .

2, 8-Dimethyl-1H-quinolin-4-one (3c): IR: 3338, 3031, $1643,1625,1569,1471,1341,733 \mathrm{~cm}^{-1}$; ${ }^{1} \mathrm{H}$ NMR (DMSO$\left.d_{6}, 300 \mathrm{MHz}\right) \delta: 2.26\left(\mathrm{~s}, 3 \mathrm{H}, \mathrm{CH}_{3}\right), 2.54\left(\mathrm{~s}, 3 \mathrm{H}, \mathrm{CH}_{3}\right), 6.08(\mathrm{~s}$, $\left.{ }^{1} \mathrm{H}, \mathrm{CH}=\right), 7.32$ (m, 1H, Ph), 7.41 (d, J=7.5 Hz, 1H, Ph), 7.68 $(\mathrm{d}, J=7.6 \mathrm{~Hz}, 1 \mathrm{H}, \mathrm{Ph}), 8.6$ (s, 1H, NH); ${ }^{13} \mathrm{C}$ NMR (DMSO- $d_{6}$, $75 \mathrm{MHz}) \delta: 17.21,24.47,104.32,116.32,124.54,127.83$, $130.25,134.43,142.20,152.35,184.54$; HRMS: calcd. for $\mathrm{C}_{11} \mathrm{H}_{11} \mathrm{NO}: 173.2753$, found: 173.6218 .

2,7-dimethyl-1H-quinolin-4-one (3d): IR: 3345, 3037, 1646, 1620, 1568, 1463, 1338, $730 \mathrm{~cm}^{-1}$; ${ }^{1} \mathrm{H}$ NMR (DMSO$\left.d_{6}, 300 \mathrm{MHz}\right) \delta: 2.21\left(\mathrm{~s}, 3 \mathrm{H}, \mathrm{CH}_{3}\right), 2.32\left(\mathrm{~s}, 3 \mathrm{H}, \mathrm{CH}_{3}\right), 6.05(\mathrm{~s}$, $1 \mathrm{H}, \mathrm{CH}=), 7.21(\mathrm{~s}, 1 \mathrm{H}, \mathrm{Ph}), 7.38(\mathrm{~d}, J=7.6 \mathrm{~Hz}, 1 \mathrm{H}, \mathrm{Ph}), 7.72$ $(\mathrm{d}, J=7.5 \mathrm{~Hz}, 1 \mathrm{H}, \mathrm{Ph}), 8.6$ (s, $1 \mathrm{H}, \mathrm{NH}) ;{ }^{13} \mathrm{C} \mathrm{NMR}$ (DMSO- $d_{6}$, $75 \mathrm{MHz}) \delta: 15.77,24.73,103.25,116.41,124.76,126.96$, 131.54, 136.38, 142.51, 156.26, 183.43; HRMS: calcd. for $\mathrm{C}_{11} \mathrm{H}_{11} \mathrm{NO}: 173.7294$, found: 173.3133 .

2-Methyl-6-methoxy-1H-quinolin-4-one (3e): IR: 3347, 3036, 1642, 1623, 1570, 1475, 1348, $743 \mathrm{~cm}^{-1}$; ${ }^{1} \mathrm{H}$ NMR $\left(\mathrm{DMSO}-d_{6}, 300 \mathrm{MHz}\right) \delta: 2.35\left(\mathrm{~s}, 3 \mathrm{H}, \mathrm{CH}_{3}\right), 3.73\left(\mathrm{~s}, 3 \mathrm{H}, \mathrm{OCH}_{3}\right)$, $6.13(\mathrm{~s}, 1 \mathrm{H}, \mathrm{CH}=), 7.45$ (d, $J=7.7 \mathrm{~Hz}, 1 \mathrm{H}, \mathrm{Ph}), 7.72(\mathrm{~d}, J=$ $7.6 \mathrm{~Hz}, 1 \mathrm{H}, \mathrm{Ph}), 7.89$ (s, 1H, Ph), 8.70 (s, 1H, NH) ${ }^{13} \mathrm{C}$ NMR $\left(\mathrm{DMSO}-d_{6}, 75 \mathrm{MHz}\right) \delta: 24.72,57.42,104.63,117.33,125.12$, 127.43, 130.46, 146.71, 154.33, 167.68, 184.15; HRMS: calcd. for $\mathrm{C}_{11} \mathrm{H}_{11} \mathrm{NO}_{2}$ : 189.3025 , found: 189.3354 .

2-Methyl-8-methoxy-1H-quinolin-4-one (3f): IR: 3342, 3039, 1641, 1631, 1573, 1478, 1341, $747 \mathrm{~cm}^{-1} ;{ }^{1} \mathrm{H}$ NMR (DMSO- $\left.d_{6}, 300 \mathrm{MHz}\right) \delta: 2.52\left(\mathrm{~s}, 3 \mathrm{H}, \mathrm{CH}_{3}\right), 3.73(\mathrm{~s}, 3 \mathrm{H}$, $\left.\mathrm{OCH}_{3}\right), 6.03(\mathrm{~s}, 1 \mathrm{H}, \mathrm{CH}=), 7.08(\mathrm{~m}, 1 \mathrm{H}, \mathrm{Ph}), 7.19(\mathrm{~d}, J=7.5$ $\mathrm{Hz}, 1 \mathrm{H}, \mathrm{Ph}), 7.53$ (d, $J=7.5 \mathrm{~Hz}, 1 \mathrm{H}, \mathrm{Ph}), 8.73$ (s, 1H, NH); ${ }^{13} \mathrm{C}$ NMR (DMSO- $\left.d_{6}, 75 \mathrm{MHz}\right) \delta: 24.13,56.21,101.48$, $116.42,122.32,124.93,125.62,132.11,148.74,167,52$, 185.71; HRMS:calcd. for $\mathrm{C}_{11} \mathrm{H}_{11} \mathrm{NO}_{2}$ : 189.2489 , found: 189.3162 .

2-Methyl-7-methoxy-1H-quinolin-4-one (3g): IR: 3351, 3034, 1647, 1633, 1575, 1471, 1358, $735 \mathrm{~cm}^{-1} ;{ }^{1} \mathrm{H}$ NMR (DMSO-d $\left.d_{6}, 300 \mathrm{MHz}\right) \delta: 2.46\left(\mathrm{~s}, 3 \mathrm{H}, \mathrm{CH}_{3}\right), 3.75\left(\mathrm{~s}, 3 \mathrm{H}, \mathrm{OCH}_{3}\right)$, $6.10(\mathrm{~s}, 1 \mathrm{H}, \mathrm{CH}=), 7.12$ (s, $1 \mathrm{H}, \mathrm{Ph}), 7.27(\mathrm{~d}, J=7.6 \mathrm{~Hz}, 1 \mathrm{H}$, $\mathrm{Ph}), 7.89$ (d, $J=7.5 \mathrm{~Hz}, 1 \mathrm{H}, \mathrm{Ph}), 8.75$ (s, 1H, NH); ${ }^{13} \mathrm{C}$ NMR (DMSO- $\left.d_{6}, 75 \mathrm{MHz}\right) \delta: 24.25,56.72,102.73,114.51,124.64$, 127.78, 131.46, 147.52, 156.21, 168,31, 186.42; HRMS: calcd. for $\mathrm{C}_{11} \mathrm{H}_{11} \mathrm{NO}_{2}$ : 189.5547 , found: 189.2396 .

2-Methyl-6-chloro-1H-quinolin-4-one (3h): IR: 3345, 3033, 1646, 1617, 1571, 1470, 1341, $704 \mathrm{~cm}^{-1}$; ${ }^{1} \mathrm{H}$ NMR (DMSO- $\left.d_{6}, 300 \mathrm{MHz}\right) \delta: 2.27\left(\mathrm{~s}, 3 \mathrm{H}, \mathrm{CH}_{3}\right), 6.17(\mathrm{~s}, 1 \mathrm{H}, \mathrm{CH}=)$, 7.31 (d, $J=7.6 \mathrm{~Hz}, 1 \mathrm{H}, \mathrm{Ph}), 7.68$ (d, $J=7.5 \mathrm{~Hz}, 1 \mathrm{H}, \mathrm{Ph}), 7.83$ $(\mathrm{s}, 1 \mathrm{H}, \mathrm{Ph}), 9.11(\mathrm{~s}, 1 \mathrm{H}, \mathrm{NH}) ;{ }^{13} \mathrm{C}$ NMR (DMSO- $\left.d_{6}, 75 \mathrm{MHz}\right)$ $\delta: 24.31,103.22,114.13,124.76,127.32,131.24,135.13$, 146.43, 151.81, 182.42; HRMS: calcd for $\mathrm{C}_{10} \mathrm{H}_{8} \mathrm{NOCl}$ : 193.8753, found: 193.6542 .

2-Methyl-8-chloro-1H-quinolin-4-one (3i): IR: 3332, 3031, 1632, 1624, 1576, 1473, 1352, $721 \mathrm{~cm}^{-1}$; ${ }^{1} \mathrm{H}$ NMR $\left(\mathrm{DMSO}-d_{6}, 300 \mathrm{MHz}\right) \delta: 2.22\left(\mathrm{~s}, 3 \mathrm{H}, \mathrm{CH}_{3}\right), 6.13(\mathrm{~s}, 1 \mathrm{H}, \mathrm{CH}=)$, $7.25(\mathrm{~m}, 1 \mathrm{H}, \mathrm{Ph}), 7.34$ (d, J=7.5 Hz, 1H, Ph), 7.52 (d, $J=7.5$ $\mathrm{Hz}, 1 \mathrm{H}, \mathrm{Ph}), 9.02$ (s, 1H, NH); ${ }^{13} \mathrm{C}$ NMR (DMSO- $d_{6}, 75 \mathrm{MHz}$ ) $\delta: 24.52,101.89,116.23,125.42,127.59,130.82,136.74$, 146.32, 153.46, 184.37; HRMS: calcd. for $\mathrm{C}_{10} \mathrm{H}_{8} \mathrm{NOCl}$ : 193.5273, found: 193.2861 .

2-Methyl-7-chloro-1H-quinolin-4-one (3j): IR: 3351, 3038, 1647, 1625, 1573, 1471, 1338, $733 \mathrm{~cm}^{-1}$; ${ }^{1} \mathrm{H}$ NMR (DMSO- $\left.d_{6}, 300 \mathrm{MHz}\right) \delta: 2.27\left(\mathrm{~s}, 3 \mathrm{H}, \mathrm{CH}_{3}\right), 6.15$ (s, $\left.1 \mathrm{H}, \mathrm{CH}=\right)$, 7.28 (s, 1H, Ph), 7.36 (d, $J=7.6 \mathrm{~Hz}, 1 \mathrm{H}, \mathrm{Ph}), 7.73$ (d, $J=7.5$ $\mathrm{Hz}, 1 \mathrm{H}, \mathrm{Ph}), 9.07$ (s, 1H, NH); ${ }^{13} \mathrm{C}$ NMR (DMSO-d 6 , $75 \mathrm{MHz}$ ) $\delta: 24.57,102.84,115.62,124.23,127.56,132.41,135.28$, 145.92, 153.64, 181.27; HRMS: calcd. for $\mathrm{C}_{10} \mathrm{H}_{8} \mathrm{NOCl}$ : 193.7468, found: 193.5396 .

2-Methyl-6-nitro-1H-quinolin-4-one (3k): IR: 3336, 3031, 1641, 1627, 1568, 1462, 1345, $732 \mathrm{~cm}^{-1}$; ${ }^{1} \mathrm{H}$ NMR (DMSO- $\left.d_{6}, 300 \mathrm{MHz}\right) \delta: 2.31\left(\mathrm{~s}, 3 \mathrm{H}, \mathrm{CH}_{3}\right), 6.12(\mathrm{~s}, 1 \mathrm{H}, \mathrm{CH}=)$, 7.42 (d, $J=7.6 \mathrm{~Hz}, 1 \mathrm{H}, \mathrm{Ph}), 7.78$ (d, J=7.8 Hz, 1H, Ph), 7.97 (s, $1 \mathrm{H}, \mathrm{Ph}), 9.13(\mathrm{~s}, 1 \mathrm{H}, \mathrm{NH}) ;{ }^{13} \mathrm{C}$ NMR (DMSO- $\left.d_{6}, 75 \mathrm{MHz}\right)$ $\delta: 24.55,104.31,114.24,125.11,128.36,132.43,135.26$, 146.35, 156.22, 186.27; HRMS: calcd. for $\mathrm{C}_{10} \mathrm{H}_{8} \mathrm{~N}_{2} \mathrm{O}_{3}$ : 204.1782, found: 204.4537 .

2-Methyl-1H-benzo[h]quinolin-4-one (31): IR: 3354, 3037, 1672, 1631, 1578, 1455, 1371, $753 \mathrm{~cm}^{-1}$; ${ }^{1} \mathrm{H}$ NMR (DMSO- $\left.d_{6}, 300 \mathrm{MHz}\right) \delta: 2.28\left(\mathrm{~s}, 3 \mathrm{H}, \mathrm{CH}_{3}\right), 6.05(\mathrm{~s}, 1 \mathrm{H}, \mathrm{CH}=)$, 7.31-7.89 (m, 6H, Ph), 8.52 (s, 1H, NH); ${ }^{13} \mathrm{C}$ NMR (DMSO$\left.d_{6}, 75 \mathrm{MHz}\right) \delta: 23.92,101.65,113.47,114.42,122.33,123.73$, $124.13,125.11,127.93,128.36,132.58,145.61,156.72$, 183.55; HRMS: calcd for $\mathrm{C}_{14} \mathrm{H}_{11} \mathrm{NO}$ : 209.3849, found: 209.7625 .

\section{RESULTS AND DISCUSSION}

Herein, we wish to report our study on the synthesis of quinoline based on Combes approach by using NKC-9 as heterogeneous catalysts. It has been observed that macroporous strongly acidic styrol resin (NKC-9), which functional group is $\mathrm{SO}_{3} \mathrm{H}$, equals to Amberlyst-15 (USA) is an efficient catalyst for construction of quinoline derivatives from anilines and ethyl acetoacetate (Fig. 1).

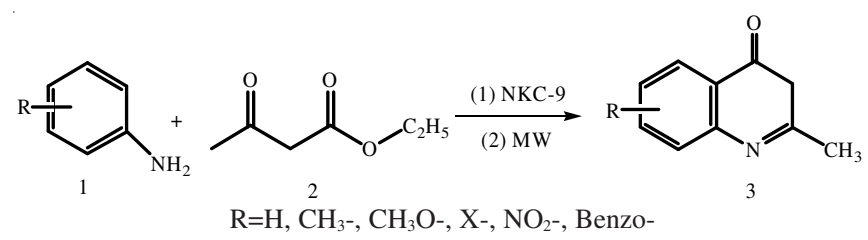

Fig. 1. Microwave Synthesis of 2-methyl-4-quinolinone under the catalyst NKC-9

Our initial study was started by reacting aniline with ethyl acetoacetate under solvent-free. The results are summarized in Table- 1 .

As shown in Table-1, the quinoline was not observed or in low yield in the absence of catalyst KNC-9 (entries 1, 2 Table-1). Prolonging reaction time could not increase quinoline yield (entries 3-6, Table-1). Augment of catalyst moderately helped to increase yields of quinoline (entries 7-9, Table-1). Inspiringly, using microwave irradiation could significantly shorten the reaction time and improve the reaction yields (entries 3-9, Table-1). 
TABLE-1

SYNTHESIS OF QUINOLINE (3a) BY REACTING ANILINE (2.0 mmol) WITHETHYL ACETOACETATE

(2.4 mmol) UNDER SOLVENT-FREE

\begin{tabular}{ccccc}
\hline Entry & NKC-9 $(\mathrm{g})$ & Time $(\min )$ & ${\text { Media }(\mathrm{SF})^{\mathrm{c}}}$ & ${\text { Yield }(3 \mathrm{a}) / \%^{\mathrm{a}, \mathrm{b}}}$ \\
\hline 1 & $/$ & 120 & r. t. & $/$ \\
2 & $/$ & 120 & $70^{\circ} \mathrm{C}$ & 53 \\
3 & 0.05 & 0.5 & $\mathrm{MW}^{\mathrm{d}}$ & 81 \\
4 & 0.05 & 1.0 & $\mathrm{MW}$ & 84 \\
5 & 0.05 & 1.5 & $\mathrm{MW}$ & 89 \\
6 & 0.05 & 2.0 & $\mathrm{MW}$ & 87 \\
7 & 0.10 & 2.0 & $\mathrm{MW}$ & 91 \\
8 & 0.15 & 2.0 & $\mathrm{MW}$ & 93 \\
9 & 0.20 & 2.0 & $\mathrm{MW}$ & 92
\end{tabular}

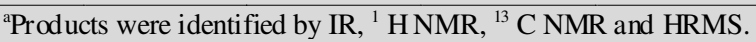

bIsolated yields.

${ }^{\mathrm{c}} \mathrm{SF}$ is Solvent-free; ${ }^{\mathrm{M}} \mathrm{MW}$ is microwave irradiation (400 w)

Furthermore, we compared reaction effect by using catalyst (NKC-9) with other reported catalysts. The results are shown in Table-2.

TABLE-2

SYNTHESIS OF QUINOLINE (3a) BY REACTING ANILINE

$(2.0 \mathrm{mmol})$ WITH ETHYL ACETOACETATE $(2.4 \mathrm{mmol})$ UNDER VARIOUS CATALYSTS

\begin{tabular}{cllc}
\hline Entry & Catalyst & \multicolumn{1}{c}{ Condition } & Yield (\%) $)^{\mathrm{a}, \mathrm{b}}$ \\
\hline 1 & $\mathrm{H}_{2} \mathrm{SO}_{4}(98 \%)$ & $\mathrm{SF} / \mathrm{MW} / 1.5 \mathrm{~min}$ & 85 \\
2 & $\mathrm{HF}$ & $\mathrm{SF} / \mathrm{MW} / 1.5 \mathrm{~min}$ & 63 \\
3 & $\mathrm{H}_{3} \mathrm{PO}_{4}$ & $\mathrm{SF} / \mathrm{MW} / 1.5 \mathrm{~min}$ & 79 \\
4 & $\mathrm{ZnCl}_{2}$ & $\mathrm{SF} / \mathrm{MW} / 1.5 \mathrm{~min}$ & 47 \\
5 & $\mathrm{SiO}_{2}$ & $\mathrm{SF} / \mathrm{MW} / 1.5 \mathrm{~min}$ & 41 \\
6 & $\mathrm{InCl}_{3}$ & $\mathrm{SF} / \mathrm{MW} / 1.5 \mathrm{~min}$ & 52 \\
7 & $\mathrm{NaHSO}_{4} / \mathrm{SiO}_{2}$ & $\mathrm{SF} / \mathrm{MW} / 1.5 \mathrm{~min}$ & 87 \\
8 & $\mathrm{NKC}_{2}$ & $\mathrm{SF} / \mathrm{MW} / 1.5 \mathrm{~min}$ & 93 \\
9 & $\mathrm{Re}-\mathrm{NKC}-9$ & $\mathrm{SF} / \mathrm{MW} / 1.5 \mathrm{~min}$ & 90 \\
\hline
\end{tabular}

${ }^{a}$ Products were identified by IR, ${ }^{1} \mathrm{HNMR},{ }^{13} \mathrm{C}$ NMR and HRMS.

IIsolated yields; ${ }^{\mathrm{C}} \mathrm{Re}-\mathrm{NKC}-9$ was regenerated resin

It was found that the yields of the reactions were not desired by using Lewis acids as catalysts (entries 4-6, Table-2). Although some proton acids could achieve high yields, they were difficult to recovery and reuse (entries 1-3, 7, Table-2). Besides high yield, our method has two prominent merits that the catalyst (NKC-9) can be recycled by using hydrochloric treatment and the reaction time is shorter (entries 8-9, Table-2).

After a comprehensive survey of the reaction conditions, acidic styrol resin (NKC-9) was considered as appropriate catalysis for Combes reaction and used to synthesize quinolines by reacting aniline $(2.0 \mathrm{mmol})$ with ethyl acetoacetate $(2.4$ mmol) under microwave irradiation $(400 \mathrm{w}, 1.5 \mathrm{~min})$ and solventfree. Subsequently, a variety of amines were examined using this method (Fig. 1). The results are listed in Table-3.

The high yields of quinolines were most obtained under such reaction conditions. It was noteworthy that electronic effect on the aniline ring strong affected on the yields of quinolines. Overall, aniline bearing electron-donating group gave higher yields of quinolines than those of electron-withdraw group. Significantly, when electron-donating groups were located at ortho- and para-aniline (entries 2, 3, 5, 6, Table-3), the reaction yields were somewhat lower than at meta-aniline (entries 4, 7, Table-3). However, the yields of quinolines were reduced when aniline carrying electron-withdraw groups (entries 8-11, Table-3), Moreover, other aromatic amines, such as naphthylamine, also could afford the corresponding quinoline under the same condition smoothly (entry 12, Table-3).

SYNTHESIS OF QUINOLINE DERIVATIVES CATALYZED
BY RESIN (NKC-9) BETWEEN AMINES AND
ETHYL ACETOACETATE

${ }^{\mathrm{a}}$ Products were identified by IR, ${ }^{1} \mathrm{H}$ NMR, ${ }^{13} \mathrm{C}$ NMR and HRMS.

${ }^{\mathrm{b}}$ Isolated yields.

\section{Conclusion}

In conclusion, we have demonstrated an efficient, mild method for the synthesis of 2-methyl-4-quinolinone by employing macroporous strongly acidic styrol resin (NKC-9) as catalyst for the Combes reaction. Furthermore, catalyst (NKC-9) can be recycled by using hydrochloric treatment. Various substituted anilines underwent the above reaction with ethyl acetoacetate to produce corresponding substituted quinolines under microwave irradiation and solvent-free. The process is operationally simple and provides excellent yields to quinolines synthesis via Combes cyclization.

\section{ACKNOWLEDGEMENTS}

The authors appreciated the financial support from Anhui Key Laboratory of Advanced Building Materials and the Natural Science Fund of Anhui Province Education Office (KJ2013A073). 


\section{REFERENCES}

1. T.R. Verhoeven, P.J. Reider, M. Labelle, J.Y. Gauthier, Y.B. Xiang and R.J. Zamboni, J. Org. Chem., 61, 3398 (1996).

2. A. Sparatore, N. Basilico, S. Parapini, S. Romeo, F. Novelli and F. Sparatorec, J. Biol. Med. Chem., 13, 5338 (2005).

3. D. Dubé, M. Blouin, C. Brideau, C.-C. Chan, S. Desmarais, D. Ethier, J.-P. Falgueyret, R.W. Friesen, M. Girard, Y. Girard, J. Guay, D. Riendeau, P. Tagari and R.N. Young, Bioorg. Med. Chem. Lett., 8, 1255 (1998).

4. T.R. Govindachari, K.R. Ravindranath and N. Viswanathan, J. Chem. Soc., Perkin Trans. I, 1215 (1974).
5. S.A. Jenekhe, L. Lu and M.M. Alam, Macromolecules, 34, 7315 (2001). 6. Z.H. Skraup, Chem. Ber. 13, 2086 (1880).

7. R. Hauser and G.A. Reynolds, J. Am. Chem. Soc., 70, 2402 (1948)

8. M. Conrad and L. Limpach, Chem. Ber, 20, 944 (1887).

9. O. Döebner and W.V. Miller, Chem. Ber., 16, 246 (1883).

10. A. Combes, Bull. Soc. Chim. Fr., 49, 89 (1888).

11. P. Friedlander, Chem. Ber., 15, 2572 (1882).

12. K. Mogilaiah, K. Vidya and T.K. Swamy, Indian J. Chem., 48B, 599 (2009).

13. B. Sapkal, K.F. Shelke, B.B. Shingate and M.S. Shingare, J. Korean Chem. Soc., 54, 723 (2010) 